\title{
Challenges in the Design and Conduct of Therapeutic Trials in Channel Disorders
}

\author{
Shannon L. Venance, ${ }^{*}$ Barbara E. Herr, ${ }^{\dagger}$ and Robert C. Griggs ${ }^{\dagger}$ \\ *University of Western Ontario, London, Ontario, Canada; ${ }^{\dagger}$ The Channelopathy Project, University of Rochester School of \\ Medicine and Dentistry, Rochester, New York 14642
}

\begin{abstract}
Summary: Neurologic channelopathies are rare, inherited paroxysmal disorders of muscle (e.g., the periodic paralyses and nondystrophic myotonias) and brain (e.g., episodic ataxias, idiopathic epilepsies, and familial hemiplegic migraine). Mutation is necessary but not sufficient for phenotypic expression and there are no simple phenotype-genotype relationships. Attacks may be spontaneous or triggered, with affected individuals often asymptomatic and neurologically normal between attacks. Performance of daily activities may be affected by the unpredictable nature; often late-onset degenerative changes cause permanent disability; for example, muscle atrophy and fixed weakness in periodic paralysis and cerebellar atrophy and progressive ataxia in the episodic ataxias. Currently, the natural history of these disorders is being defined. Clearly, the estab-
\end{abstract}

lished methodologies for randomized controlled clinical trials are not feasible for rare diseases and innovative trial design is essential. There is a requirement for clinically relevant outcome measures for episodic disorders. Increasing our knowledge of the pathophysiology will help in targeting and designing rational therapeutic approaches. We will use the current understanding of the neurological channelopathies to illustrate some of the opportunities, challenges, and strategies in bringing safe and effective treatments to patients. There are reasons for optimism that new partnerships between clinical investigators, government, patient advocacy groups, and industry will prevent symptoms and progression of the neurological channelopathies. Key Words: Neurological channelopathies, trial design, outcome measures, rare diseases, n-of-1 studies.

\section{INTRODUCTION}

Neurologic channelopathies, caused by mutations in voltage-gated and ligand-gated ion channel genes, are rare paroxysmal disorders affecting excitable cells of the nervous system from skeletal muscle to brain (Table 1). Importantly, mutations in these channel genes are necessary but not sufficient for phenotypic expression, and affected individuals are often asymptomatic and neurologically normal between attacks. These channel disorders share common features, including the episodic nature of attacks that are often triggered (for example, by stress, exercise, hormones) and an onset in childhood or adolescence. Performance at school, home, and work may be affected by unpredictable episodes. Additionally, there may be late-onset degenerative changes resulting in permanent disability; for example, muscle atrophy and

Address correspondence and reprint requests reprints to: Shannon L. Venance, MD, PhD, Department of Clinical Neurological Sciences, London Health Sciences Centre, 339 Windermere Road, C7-131, London, ON N6A 5A5, Canada. E-mail: svenanc2@uwo.ca. fixed proximal weakness in periodic paralysis (PP) or cerebellar atrophy, and progressive ataxia in the episodic ataxias (EA). Could the cumulative attacks cause progressive injury to affected tissues over time (the brain in epilepsy; the cerebellum in EA; muscle in PP and the nondystrophic myotonias)? Will prevention of attacks prevent the development of permanent deficits? ${ }^{1,2}$ While it is clear that treatment is important, should treatment efforts be directed at the molecular lesion, the precipitating factors, or both?

The breakthroughs on the electrophysiological characterization of ion channel function ${ }^{3}$ and the molecular genetics of the neurological channelopathies ${ }^{4}$ offer exciting opportunities for accurate diagnosis and rational, perhaps even individually-tailored therapy based on genotype and pathophysiology. Do we need to temper our optimism in the face of challenges in carrying out clinical trials? We will use our current understanding of the neurological channelopathies to illustrate some of the opportunities, challenges, and strategies that lie ahead in bringing safe and effective treatments to our patients. 
Table 1. Examples of Neurological Channelopathies

\begin{tabular}{|c|c|c|}
\hline Phenotype & Gene & Channel \\
\hline \multicolumn{3}{|l|}{ Skeletal Muscle } \\
\hline НypoPP1 & CACNA1S & Calcium, $\alpha$ subunit \\
\hline HyроРP2 & $S C N 4 A$ & Sodium, $\alpha$ subunit \\
\hline HyperPP & $S C N 4 A$ & Sodium, $\alpha$ subunit \\
\hline PMC & $S C N 4 A$ & Sodium, $\alpha$ subunit \\
\hline PAM & $S C N 4 A$ & Sodium, $\alpha$ subunit \\
\hline ATS & $K C N J 2$ & Potassium \\
\hline $\mathrm{MC}$ & CLCN1 & Chloride \\
\hline CMS & CHRNA & Nicotinic Ach receptor, $\alpha$ subunit \\
\hline CMS & $C H R N B$ & Nicotinic Ach receptor, $\beta$ subunit \\
\hline CMS & CHRNE & Nicotinic Ach receptor, $\epsilon$ subunit \\
\hline CMS & $S C N 4 A$ & Sodium, $\alpha$ subunit \\
\hline \multicolumn{3}{|l|}{ Peripheral Nerve } \\
\hline FPE & $S C N 9 A$ & Sodium, $\alpha$ subunit \\
\hline \multicolumn{3}{|l|}{ Central Nervous System } \\
\hline Hyperekplexia & GLRA1 & Glycine receptor \\
\hline EA1 & KCNAI & Potassium \\
\hline EA2 & CACNA1A & Calcium, $\alpha$ subunit \\
\hline FHM1 & CACNAIA & Calcium, $\alpha$ subunit \\
\hline FHM2 & ATP1A2 & Na-K-ATPase \\
\hline FHM3 & SCN1A & Sodium, $\alpha$ subunit \\
\hline SCA 6 & CACNA1A & Calcium, $\alpha$ subunit \\
\hline $\mathrm{BNFC}$ & $K C N Q^{2}$ & Potassium \\
\hline BNFC & $K C N Q 3$ & Potassium \\
\hline ADNFLE & CHRNA4 & $\begin{array}{l}\text { Nicotinic Ach receptor, } \alpha_{4} \\
\text { subunit }\end{array}$ \\
\hline ADNFLE & CHRNB2 & $\begin{array}{l}\text { Nicotinic Ach receptor, } \beta_{2} \\
\text { subunit }\end{array}$ \\
\hline GEFS + & $S C N 1 B$ & Sodium, $\beta_{1}$ subunit \\
\hline $\mathrm{GEFS}+/ \mathrm{BFNC}$ & $S C N 2 A$ & Sodium, $\alpha_{2}$ subunit \\
\hline GEFS +/ICEGTC/SMEI & SCN1A & Sodium, $\alpha_{1}$ subunit \\
\hline $\mathrm{GEFS}+/ \mathrm{FS} / \mathrm{CAE}$ & $G A B R G 2$ & $\mathrm{GABA}_{\mathrm{A}}$ receptor, $\gamma_{2}$ subunit \\
\hline ADJME & GABRAl & $\mathrm{GABA}_{\mathrm{A}}$ receptor, $\alpha_{1}$ subunit \\
\hline GEPD & KCNMA1 & $\mathrm{BK}$ potassium \\
\hline CAE/JAE/JME & CLCN2 & Chloride \\
\hline
\end{tabular}

ADJME, autosomal dominant juvenile myoclonic epilepsy; ADNFLE, autosomal dominant nocturnal frontal lobe epilepsy; ATS, AndersenTawil syndrome; BNFC, benign neonatal familial convulsions; CAE, childhood absence epilepsy; CMS. congenital myasthenic syndrome; EA, episodic ataxia; FHM, familial hemiplegic migraine; FPE, familial primary erythermalgia; FS, febrile seizures; GEFS +, generalized epilepsy with febrile seizures+; GEPD, generalized epilepsy with paroxysmal dyskinesias; HyperPP, hyperkalemic periodic paralysis; HypoPP, hypokalemic periodic paralysis; ICEGTC, intractable childhood epilepsy with frequent generalized tonic-clonic seizures; JAE, juvenile absence epilepsy; JME, juvenile myoclonic epilepsy; MC, myotonia congenita; PAM, potassium aggravated myotonia; PMC, paramyotonia congenita; SCA6, spinocerebellar ataxia; SMEI, severe myoclonic epilepsy of infancy.

\section{PHILOSOPHICAL CONSIDERATIONS}

The design and conduct of clinical trials is a major undertaking even in common diseases that affect large numbers of individuals. In the genomic era, some common diseases could be considered rare, given the spectrum of mutations; conducting therapeutic trials in rare hereditary disorders adds yet another level of complexity. For example, Duchenne dystrophy (DD) is a common and well characterized clinical disease, and yet so far there are no currently approved therapies for DD despite a number of well designed clinical trials. ${ }^{5}$ Identification of the molecular lesion in DD caused much anticipation for targeted therapeutic strategies. However, it has become apparent that there are many different, often private, mutations occurring within a large number of exons in the dystrophin gene; from a therapeutic perspective, DD could be considered many somewhat different diseases. Neurological channelopathies, like the majority of rare diseases, are primarily genetic in nature. Pursuing experimental therapeutics in the monogenic channelopathies will continue to provide insight into normal physiology and will further our understanding of more common channel diseases such as migraine, epilepsy, and myotonic dystrophy.

\section{ON CLINICAL TRIAL DESIGN IN GENERAL}

There are many established clinical trial methodologies. Many trials require costly, multicenter collaborative efforts in order to accumulate sufficient numbers of subjects to detect significant treatment effects in common diseases. 
Given the same requirement to demonstrate safety and efficacy in an ethical manner in any rare disease, the challenge of conducting randomized, double-blind, controlled clinical trials (RCT) may seem insurmountable. Depending on the power calculation, the recruitment phase may be problematic and enrollment protracted over years (if not decades) for the treatment effect to reach significance and change clinical practice. ${ }^{5}$

Furthermore, there has been little incentive for pharmaceutical companies to become engaged in rare disease clinical research because little financial gain would be expected with a limited market. However, concerted efforts by patient advocacy groups to increase awareness of rare diseases were instrumental in pushing forward legislation such as the U.S. Orphan Drug Act of $1982^{6}$ and the European Orphan Drug Regulation, ${ }^{7}$ which provide government incentives (such as tax credits and periods of market exclusivity) to encourage industry to invest in developing and marketing therapies for rare diseases such as the neurological channelopathies. Importantly, orphan drug status can apply not only to new drug development (which remains expensive) but also to establishing a novel indication for a currently (or previously) marketed drug, such as the carbonic anhydrase inhibitors in periodic paralysis ${ }^{8}$ and mexiletine in myotonia. ${ }^{9}$

Proposed alternatives to parallel group RCTs for rare diseases include blinded crossover designs, placebo-controlled n-of-1 studies, or natural history controlled trials. In crossover studies, each participant acts as his or her own control and receives the study treatments sequentially in a randomly determined order. This design reduces the sample size needed for adequate power compared with a parallel group design, provided close attention is paid to potential period and carryover effects, blinding, and dropouts during the design phase of the trial. ${ }^{10}$

$\mathrm{N}$-of-1 trials may have direct appeal to patients. They are directly applicable to the participant; they avoid the heterogeneity of effect in the larger RCT, and they permit patients to be actively involved in decisions of benefit versus adverse reaction. ${ }^{5}$ An n-of- 1 clinical trial uses randomization, blinding, and reliable endpoints, but the outcome (beneficial or not) is specific to that individual. ${ }^{11}$ In other words, an n-of-1 trial is an RCT for one individual. The study treatment-intervention and placebo-comparator are administered in a randomized order with the outcome(s) assessed in a blinded manner. The trial will consist of repeated paired treatment cycles (each cycle a separate randomization of the allocation order for intervention and placebo) until it becomes evident whether the intervention is or is not effective. ${ }^{11,12}$ Multiple n-of-1 clinical trials may be combined to estimate population effects, similar to a crossover trial. ${ }^{12}$

While these alternatives to parallel group RCT are attractive, careful trial design remains necessary as potential pitfalls exist for conducting therapeutic trials in neurological channelopathies. For example, it is important to ensure a sufficient washout interval between periods in crossover and n-of-1 trials to avoid carryover effects from the intervention in the initial period that may bias the results in the following period. The duration of the treatment periods must be appropriate to detect a change in the outcome variable (e.g., number of episodes) in the participants as it is well recognized that the frequency of attacks (periodic paralysis) or seizures (epilepsy) may be highly variable (ranging from daily to annually). Natural history controlled studies are not currently feasible for the neurological channel disorders; there are no prospectively collected, standardized databases available (see below). Potential challenges in using historical controls for future channelopathy trials include clinical variability and selection bias, in particular if data collection and diagnostic standards are not standardized.

As previously mentioned, another challenge to overcome is the need for detailed natural history data. ${ }^{8}$ Natural history data will allow investigators to calculate sample size, to identify meaningful outcome variables for clinical trials and, perhaps more importantly, to understand what may represent clinically important change. Fortunately, clinical research networks in North America and Europe have come together for the specific purpose of studying rare diseases in a standardized manner (Rare Diseases Clinical Research Network [RDCRN]: www.rarediseasesnetwork.org and European Clinical Research Infrastructures Network [ECRIN]: www. ecrin.org). The neurological channel disorders being studied under the RDCRN umbrella include the episodic ataxias, the nondystrophic myotonias, and the AndersenTawil syndrome.

Patient advocacy groups are integral members of these clinical networks, as it remains vital to seek out what is important to patients when considering what constitutes significant change. There are a number of validated instruments assessing patient satisfaction with treatments, as well as generic and specific instruments for measuring health-related quality of life (HRQOL). Generic instruments allow comparisons across disease groups whereas specific instruments target a particular group, disease, or intervention. It has been shown, at least for RCT in which a therapeutic effect was demonstrated, that specific instruments are more responsive than generic tools, supporting the idea that a combination of specific and generic instruments are useful. ${ }^{13}$ While there are specific HRQOL instruments for several neurological diseases (e.g., epilepsy, migraine, and neuromuscular disorders), none specifically address function during an episode of illness (J. Langfitt, personal communication).

\section{ISSUES IN CLINICAL TRIALS FOR NEUROLOGICAL CHANNEL DISORDERS}

\section{Current treatments}

Many of the channel disorders have treatments that are believed to be effective. These observations principally 
arose from clinicians treating individual patients and observing their response. Anecdotal evidence and small clinical series support the use of acetazolamide in periodic paralysis or episodic ataxia and mexiletine in myotonia. Few treatments have been rigorously studied in a randomized, double-blind fashion with the exception of dichlorphenamide in periodic paralysis. ${ }^{14}$

\section{Phenotype and genotype}

It is becoming clear that simple phenotype-genotype relationships are the exception; phenotypes are increasingly viewed as complex evolving properties influenced by dynamically changing environments and are not easily predicted..$^{15}$ Andersen-Tawil syndrome, ${ }^{16,17}$ episodic ataxia, ${ }^{18-20}$ and the inherited epilepsies ${ }^{21,22}$ demonstrate marked intra- and interfamilial phenotypic variability; mutations do not predict phenotype. For example, the monogenic epilepsies cannot be distinguished by clinical features from the more common and polygenic epilep$\operatorname{sies}^{23}$ and we cannot predict recessive or dominant inheritance in the nondystrophic myotonias based on the location or type of mutation in CLCN1. ${ }^{24}$ This variability strongly suggests that additional modifying factors, environmental or genetic, have yet to be identified. Prospective, standardized natural history data such as demographics, including gender, response to treatment, and electrophysiology, may identify unique or unusual features that may help direct future research.

Many of the channel disorders display locus heterogeneity (see Table 1) despite clinically indistinguishable phenotypes; for example, hypokalemic periodic paralysis (hypoPP) due to mutations in CACNAIS and SCN4A, autosomal dominant nocturnal frontal lobe epilepsy caused by mutations in CHRNA4 and CHRNB2, and familial hemiplegic migraine caused by mutations in CACNA1A, SCNIA, and ATPIA2. Moreover, mutations in a single gene cause clinically distinct allelic disorders (SCN4A mutations causing hyperkalemic periodic paralysis [hyperPP], hypoPP, paramyotonia congenita, potassium aggravated myotonia, and congenital myasthenic syndrome, or CACNA1A causing EA2, familial hemiplegic migraine and spinocerebellar ataxia 6). Unfortunately, genetic testing on a clinical basis is not yet widely available for the channel disorders, and in particular for large genes with primarily private mutations (for example CLCN1 mutations in myotonia congenita).

Accurate genotyping is of critical importance to permit precise classification of affected individuals in natural history studies, to stratify participants for therapeutic trials, and to understand response to treatments in future trials. For instance, there is well described variable responsiveness to acetazolamide treatment in patients with EA. ${ }^{18}$ Patients with hypoPP and mutation in SCN $4 A$ were excluded from the dichlorphenamide clinical trial because of individual reports of worsening with another carbonic anhydrase inhibitor, acetazolamide (ACZ). ${ }^{14}$ There are, however, case reports of patients with SCN4A mutations that respond well to ACZ. ${ }^{25,26}$ Congenital myasthenic syndromes (CMS) are caused by loss-of-function of presynaptic, synaptic, and postsynaptic proteins at the neuromuscular junction ${ }^{27}$ with implications for treatment. ${ }^{28}$ For example, some individuals with a postsynaptic slow channel CMS syndrome did not respond to acetylcholinesterase inhibition but were reported to respond to fluoxetine. ${ }^{29}$

\section{Outcome measures}

While each of the neurological channelopathies has a distinct clinical presentation (stiffness, weakness, headache, incoordination, seizure), there are common features as well as overlap. Discrete episodes may be spontaneous or triggered in all (stress, exercise, rest after exercise, hormones, etc). If we can understand how the triggers precipitate attacks, we may find opportunity for intervention. One of the challenges in clinical trial design with episodic disorders is selecting relevant outcome measures in the absence of standardized historical databases, and as noted above, where none of the HRQOL instruments specifically addresses the paroxysmal nature of these disorders.

Attack frequency, duration, and severity are outcome variables that rely on self-report. If attack frequency is selected, then only individuals with a "minimum" number of episodes sufficient to allow detection of a change over the duration of the trial would be eligible. How do we capture the data comprehensively and reliably? Paper diaries were used successfully in the DCP trial in periodic paralysis $;{ }^{14}$ however, the diaries were often not received in a timely manner and were sometimes difficult to read. ${ }^{30}$ Potential drawbacks to paper diaries have been recognized in other studies, and have recently led to the development of electronic methods. ${ }^{31,32}$

A study that measured compliance of patients with chronic pain, using paper or electronic diaries, showed subjects completed $90 \%$ of the diaries. Participants in the study were asked to make three entries daily at set times in either paper or electronic diaries. The paper diaries were designed to monitor the date of entries, but the subjects did not know this. Subjects knew that the electronic diaries were timestamped. Seventy percent of the paper data was filled in retrospectively (termed "data hoarding"). Some diaries were even completed prospectively ("data predicting"). In contrast, there was 94\% compliance with the electronic diaries. ${ }^{33}$ Pain studies also indicate that clinical data that are self-reported retrospectively are biased by the severity of current symptoms. ${ }^{34}$ Therefore, timely capture is important. Electronic diaries have been successfully used in recent Federal Drug Administration approved indications for new drugs for treatment of asthma, attention deficit hyperactivity disorder, and daytime sleepiness (P. Eisenmann, PHT Corporation, personal communication). 
Data can be electronically captured through hand-held computers or through interactive voice response (IVR) using the telephone. IVR is currently being tested in the $R D C R N .^{35}$ A planned National Institutes of Health trial of acetazolamide and dichlorphenamide in the periodic paralyses uses a hand-held commercial electronic device that transmits the data each night via the subject's phone line and is compliant with all international clinical trial regulations. ${ }^{30}$ Technology alone cannot achieve accurate self-report. Frequent interaction between subjects and investigators is necessary to educate the subjects in the study definition of "attack" or "episode" and to maintain the participants' motivation to make timely and complete reports of episode intensity and duration. This training is critical. For example, prior to training, a highly educated patient may report having had no episodes in a month, and only with direct questioning reveal that there had been problems arising in the morning and that sometimes one limb felt weak.

A relatively recent opportunity is using electrodiagnostic evaluation of muscle membrane excitability as a surrogate measure in the skeletal muscle channelopathies. ${ }^{36}$ Standardized short and long exercise protocols, in addition to routine nerve conduction studies and needle electromyography, were studied in patients with chloride, calcium, and sodium channelopathies. ${ }^{37,38}$ The protocols helped to define discrete electromyographic patterns that correlated with channel mutations and may be valuable as clinical outcomes in therapeutic trials, although further research is needed to define responsiveness to treatment.

\section{PATHOPHYSIOLOGY}

A lengthy, demanding, and expensive process exists for drug development when seeking regulatory approval and involves in vitro and preclinical animal studies in addition to the human clinical trials. ${ }^{6}$ Partnerships between clinical investigators, industry, government, and patient advocacy groups are in place and are necessary to advance experimental therapeutics in channelopathies, whether targeting a disease mechanism or an individual genomic profile. Understanding the molecular biology and pathophysiology of the disease process hopefully leads to the rational targeting of therapeutics. An illustration of this concept is the report of response to 4-aminopyridine ( $5 \mathrm{mg}$ three times a day) in three patients with EA-2. ${ }^{39}$ Treatment was based on the hypothesis that reduced calcium current in EA-2 leads to disinhibition of cerebellar Purkinje cells and that potassium channel blockers may increase Purkinje cell inhibitory influences. A prospective clinical trial is currently in the planning stages to confirm this observation.

However, this orderly sequence of events (identification of a molecular defect, unraveling the pathophysiology, completion of preclinical studies, and implementation of clinical trials) rarely holds true. Fortunately, the process need not be completed in sequential fashion. The potassium or M-current conductance through KCNQ2 and KCNQ3 channels has an important inhibitory role in regulation of the firing rate of neurons; relatively small reductions in the M-current predispose to neuronal hyperexcitability. ${ }^{15,40} \mathrm{Re}-$ tigabine (currently in phase II trials for refractory partial epilepsy) was known to activate potassium current in vitro and have anticonvulsant properties in animal models well before it was recognized as specific for the KCNQ M-type potassium current, which is reduced in benign neonatal familial convulsions. ${ }^{41}$

In the CNS, the ion channels identified to date suggest molecular targets for therapeutic trials; however, we still lack complete understanding of pathophysiology. Why mutations in the nicotinic acetylcholine receptor, widely expressed in the brain, should cause focal onset epilepsy originating in the frontal lobe remains obscure. ${ }^{23}$ The $\beta_{1}$ subunit of the sodium channel is ubiquitously expressed in the electrically excitable tissues of brain, heart, and skeletal muscle, yet mutations in $S N C 1 B$ cause generalized epilepsy with febrile seizures + without paroxysmal symptoms in heart or muscle. ${ }^{24}$ The difficulty lies in the absence of explanations as to how interruption of a particular channel affects complex neuronal circuitry. In vitro and animal models will be essential as seizure semiology may be common to several different ion channel mutations and interictal electroencephalogram changes are nonspecific. ${ }^{21}$

On the other hand, the skeletal muscle channelopathies provide accessible and relatively well understood electrophysiology. Yet, even here there is work to be done. For example, the pathophysiology of hyperkalemic periodic paralysis is reasonably clear but not so with hypoPP, ${ }^{24}$ and yet ACZ is an effective therapy for many individuals affected with either hyperPP or hypoPP. ${ }^{8}$ Similarly, dichlorphenamide, another carbonic anhydrase inhibitor, reduced attack frequency and severity in both of the periodic paralyses. ${ }^{14}$ Why carbonic anhydrase inhibitors should be effective in periodic paralysis has been a subject of research and debate. In vitro studies have suggested activation of calcium-activated potassium channels rather than carbonic anhydrase inhibition. ${ }^{42}$ More recently, it has been proposed that ACZ modulates chloride channel function via intracellular acidification, enhancing chloride conductance at the membrane resting potential, which would oppose the pathologic depolarizing currents seen in the PP. ${ }^{43}$

\section{CONCLUSIONS}

The future remains bright for experimental therapeutics in neurological channel diseases. Despite the challenges that lie ahead, there are many exciting opportunities. New partnerships among clinical investigators, industry, government, and patient advocacy groups are moving the rare disease field forward. Geographically dispersed clinical research networks will characterize 
natural history, identify relevant outcome measures, and move ahead with innovative trial designs for therapeutic trials in neurological channel disorders.

Acknowledgments: Studies were supported in part by a General Clinical Research Center (GCRC) grant, 5 M01 RR00044 from the National Center for Research Resources, NIH; studies were funded by 8U54 NS059065, from the National Institute of Neurological Disorders and Stroke, and under the aegis of the Office of Rare Diseases and the National Center for Research Resources (NCRR), components of the National Institutes of Health (NIH). The contents are solely the responsibility of the authors and do not necessarily represent the official views of NCRR or NIH.

\section{REFERENCES}

1. Griggs RC, Engel WK, Resnick JS. Acetazolamide treatment of hypokalemic periodic paralysis. Prevention of attacks and improvement of persistent weakness. Ann Intern Med 1970;73:3948.

2. Dalakas MC, Engel WK. Treatment of "permanent" muscle weakness in familial hypokalemic periodic paralysis. Muscle Nerve 1983;6:182-186.

3. Tawil R, Cannon S. Neurologic channelopothies: Evolving concepts and therapeutic challenges. Neurotherapeutics 2007;4:173.

4. Cannon SC. Physiologic principles underlying ion channelopothies. Neurotherapeutics 2007;4:174-183.

5. Griggs RC. Developing new treatments for muscle disease: prospects and promise. Curr Opin Neurol 1994;7:422-426.

6. Haffner ME. Adopting orphan drugs--two dozen years of treating rare diseases. N Engl J Med 2006;354:445-447.

7. Wastfelt M, Fadeel B, Henter J-I. A journey of hope: lessons learned from studies on rare diseases and orphan drugs. J Intern Med 2006;260:1-10.

8. Venance SL, Cannon SC, Fialho D, et al. The primary periodic paralyses: diagnosis, pathogenesis and treatment. Brain 2006;129: $8-17$.

9. Moxley RT III. Channelopathies. Curr Treat Options Neurol 2000; 2:31-47.

10. Lagakos SW. Clinical trials and rare diseases. N Engl J Med 2003;348:2455-2456.

11. Guyatt G, Sackett D, Taylor DW, Chong J, Roberts R, Pugsley S. Determining optimal therapy-randomized trials in individual patients. N Engl J Med 1986;314:889-892.

12. Sung L, Feldman BM. N-of-1 trials: innovative methods to evaluate complementary and alternative medicines in pediatric cancer. J Pediatr Hematol Oncol 2006;28:263-266.

13. Wiebe S, Guyatt G, Weaver B, Matijevic S, Sidwell C. Comparative responsiveness of generic and specific quality-of-life instruments. J Clin Epidemiol 2003;56:52-60.

14. Tawil R, McDermott MP, Brown R Jr, et al. Randomized trials of dichlorphenamide in the periodic paralyses. Working Group on Periodic Paralysis. Ann Neurol 2000;47:46-53.

15. Burgess DL. Neonatal epilepsy syndromes and GEFS +: mechanistic considerations. Epilepsia 2005;46(suppl 10):51-58.

16. Sansone V, Griggs RC, Meola G, et al. Andersen's syndrome: a distinct periodic paralysis. Ann Neurol 1997;42:305-312.

17. Sansone V, Tawil R. Management and treatment of AndersenTawil Syndrome (ATS). Neurotherapeutics 2007;4:233-237.

18. Jen J, Kim GW, Baloh RW. Clinical spectrum of episodic ataxia type 2. Neurology 2004;62:17-22.

19. Rajakulendran S, Schorge S, Kullman DM, Hanna MG. Episodic ataxia type 1: Neuronal potassium channelopathy. Neurotherapeutics $2007 ; 4: 258-266$.
20. Strupp M, Zwergal A, Brandt T. Episodic ataxia type 2. Neurotherapeutics 2007;4:267-273.

21. Heron SE, Scheffer IE, Berkovic SF, Dibbens LM, Mulley JC. Channelopathies in idiopathic epilepsy. Neurotherapeutics 2007; 4: 295-304.

22. Vernino S. Autoimmune and paraneoplastic channelopathies. Neurotherapeutics 2007;4:305-314.

23. Steinlein OK. Genetic mechanisms that underlie epilepsy. Nat Rev Neurosci 2004;5:400-408.

24. Cannon SC. Pathomechanisms in channelopathies of skeletal muscle and brain. Annu Rev Neurosci 2006;29:387-415.

25. Venance SL, Jurkat-Rott K, Lehmann-Horn F, Tawil R. SCN4Aassociated hypokalemic periodic paralysis merits a trial of acetazolamide. Neurology 2004;63:1977.

26. Vicart S, Sternberg D, Fournier E, et al. New mutations of SCN4A cause a potassium-sensitive normokalemic periodic paralysis. Neurology 2004;63:2120-2127.

27. Engel AG. The therapy of congenital myasthenic syndromes. Neurotherapeutics 2007;4:252-257.

28. Jurkat-Rott K, Lehmann-Horn F. Muscle channelopathies and critical points in functional and genetic studies. J Clin Invest 2005; 115:2000-2009.

29. Harper CM, Fukodome T, Engel AG. Treatment of slow-channel congenital myasthenic syndrome with fluoxetine. Neurology 2003; 60:1710-1713

30. Herr B, Kamp C, Trimble G, et al. Utility of electronic self-report tool to measure primary outcome in the HYP HOP study. Paper presented at: International Periodic Paralysis Meeting; October 2004; Rochester, NY.

31. Henker B, Whalen CK, Jamner LD, Delfino RJ. Anxiety, affect, and activity in teenagers: monitoring daily life with electronic diaries. J Am Acad Child Adolesc Psychiatry 2002;41:660-670.

32. Kerkenbush NL, Lasome CE. The emerging role of electronic diaries in the management of diabetes mellitus. AACN Clin Issues 2003; 14:371-378.

33. Stone AA, Shiffman S, Schwartz JE, Broderick JE, Huffard MR. Patient compliance with paper and electronic diaries. Control Clin Trials 2003;24:182-199.

34. Eich E, Reeves J, Jaeger B, Graff-Radford S. Memory for pain: relation between past and present pain intensity. Pain 1985;23: 375-379.

35. Herr BE, Malloy J, Cleland J, Krischer J. Automated tools for data collection and management in clinical research studies of Andersen-Tawil syndrome: improving protocol compliance and data quality. Paper presented at: National Leaders Forum; May 31-June 1, 2006; Rockville, MD.

36. Cleland JC, Logigian EL. Clinical evaluation of memebrane excitability in muscle channel disorders: Potential applications in clinical trials. Neurotherapeutics 2007;4:205-215.

37. Fournier E, Arzel M, Sternberg D, et al. Electromyography guides toward subgroups of mutations in muscle channelopathies. Ann Neurol 2004;56:650-661.

38. Fournier E, Viala K, Gervais H, et al. Cold extends electromyography distinction between ion channel mutations causing myotonia. Ann Neurol 2006;60:356-365.

39. Strupp M, Kalla R, Dichgans M, Freilinger T, Glasauer S, Brandt $\mathrm{T}$. Treatment of episodic ataxia type 2 with the potassium channel blocker 4-aminopyridine. Neurology 2004;62:1623-1625.

40. Lerche H, Jurkat-Rott K, Lehmann-Horn F. Ion channels and epilepsy. Am J Med Genet 2001;106:146-159.

41. Rogawski MA. Diverse mechanisms of antiepileptic drugs in the development pipeline. Epilepsy Res 2006;69:273-294.

42. Tricarico D, Barbieri M, Mele A, Carbonara G, Camerino DC. Carbonic anhydrase inhibitors are specific openers of skeletal muscle BK channel of K+-deficient rats. FASEB J 2004;18:760-761.

43. Eguchi H, Tsujino A, Kaibara M, et al. Acetazolamide acts directly on the human skeletal muscle chloride channel. Muscle Nerve 2006;34:292-297. 